\title{
Suggested Exercise Protocol for High Risk Population with COVID-19
}

\author{
Hala I. Awadalla*, Mostafa A. Abou Shokka, Nadia F. Deyab \\ Medical Science Department, Institute of Environmental Studies and Research, Ain Shams University, Cairo, Egypt \\ Email: *Hala_Awadalla@yahoo.com
}

How to cite this paper: Awadalla, H.I., Shokka, M.A.A. and Deyab, N.F. (2020) Suggested Exercise Protocol for High Risk Population with COVID-19. Open Journal of Rheumatology and Autoimmune Diseases, 10, 146-156.

https://doi.org/10.4236/ojra.2020.103016

Received: July 11, 2020

Accepted: August 15, 2020

Published: August 18, 2020

Copyright $\odot 2020$ by author(s) and Scientific Research Publishing Inc. This work is licensed under the Creative Commons Attribution International License (CC BY 4.0).

http://creativecommons.org/licenses/by/4.0/

\begin{abstract}
Background: People who have underlying medical conditions and those over 60 years old have a higher risk of developing coronavirus disease complications (COVID-19) with more mortality rate. Breathing exercises is good for clearing the mucus from lungs and improves chest expansion while circulatory exercises enhance cardiopulmonary/vascular systems. Aim: This study aims to suggest exercise program for prevention, decrease morbidity and mortality in COVID-19 patients and patients with other respiratory co-morbidities. Conclusion: Regular simple exercises include respiratory and circulatory exercises may have a great effect on improving lung function and immunity in addition to relieving psychological stress thus can help in treatment of high risk people from COVID-19.
\end{abstract}

\section{Keywords}

COVID-19, Pneumonia, Geriatrics, Breathing Exercises, Circulatory Exercises

\section{Introduction}

Coronavirus disease is an infectious disease caused by a newly discovered (COVID19) coronavirus. COVID-19 can result in severe disease, including hospitalization, admission to an intensive care unit, and death [1]. Most people who get COVID-19 experience mild to moderate symptoms like coughing and high body temperature, or fever, these people generally recover. Other people, the virus gets deep into the lungs and causes a severe infection: pneumonia. Although most human coronavirus infections are mild, the epidemics of the two beta coronaviruses, Severe Acute Respiratory Syndrome coronavirus (SARS-CoV) and Middle East Respiratory Syndrome coronavirus (MERS-CoV), have caused more than 10,000 cumulative cases in the past two decades, with mortality rates of 
$10 \%$ for SARS-CoV and 37\% for MERS-CoV [2].

Older people and those with underlying medical problems like cardiovascular disease, diabetes, chronic respiratory disease, and cancer are more likely to develop serious illness by COVID-19 [3].

Histologically, COVID-19 causes diffuse alveolar damage (DAD) with pronounced pulmonary edema andhyaline membrane formation (Figure 1). In some areas, there is interstitial thickening, with mild to moderate fibrosis, but a disproportionately sparse infiltrate of inflammatory cells (mainly histiocytes, including multinucleated forms, and lymphocytes) [4].

Although the clinical manifestations of COVID-19 are dominated by respiratory symptoms, some patients have severe cardiovascular damage. Angiotensinconverting enzyme 2 (ACE2) is involved in heart function and the development of hypertension and diabetes mellitus. In addition, ACE2 has been identified as a functional receptor for coronaviruses, including SARS-CoV and SARS-CoV-2. SARS-CoV-2 infection is triggered by binding of the spike protein of the virus to ACE2, which is highly expressed in the heart and lungs. Reports suggest that MERS-CoV can cause acute myocarditis and heart failure. SARS-CoV-2 and MERS-CoV have similar pathogenicity, and the myocardial damage caused by infection with these viruses [5].

It is widely accepted that regular physical activity is beneficial for cardiovascular health. Frequent exercise is robustly associated with a decrease in cardiovascular mortalityas well as the risk of developing cardiovascular diseases. Physically active individuals have lower blood pressure, higher insulin sensitivity, and a more favorable plasma lipoprotein profile. Exercise has also been found to have beneficial effects on the heart. Acutely, exercise increases cardiac output and blood pressure, but individuals adapted to exercise show lower resting heart rate and blood pressure [6]. The respiration system consists of organs responsible for taking in oxygen for respiration and releasing carbon dioxide and water vapor, which are the waste products formed during respiration. The passages in the nose, windpipe (trachea), bronchi, lungs and air sacs are the main organs of

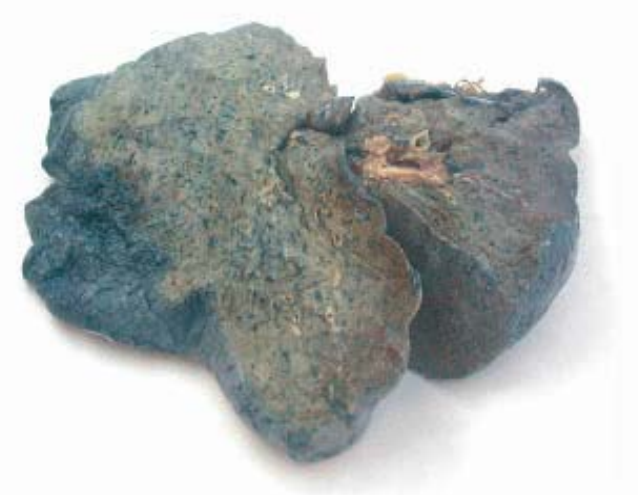

Figure 1. Histological features of lungs in patients with severe acute respiratory syndrome. Extensive consolidation with a grayish cut surface was noted in most of the patients [4]. 
the respiratory system. Many parameters of respiratory system get affected due to physical exercises [7].

New England Complex Systems Institute stated that improving respiratory health even before becoming infected, good ventilation, and frequent cleaning, of the environment of individuals that are ill by COVID-19 is widely recommended to improve outcomes [8].

World health organization (WHO) in $18^{\text {th }}$ of March (2020) applied messages for older adults, people with underlying health condition to learn simple daily physical exercises to perform at home, in quarantine or isolation so can maintain mobility and reduce boredom, while they applied messages for people in isolation to exercise regularly to improve mental health and psychosocial considerations during theCOVID-19 outbreak [9].

Chigira et al. (2015) [10] performed early physiotherapy for elderly patients with pneumonia admitted to an ICU and examined its influence on the severity of pneumonia. They compared the results of the standard and early intervention groups. In the early intervention group, the ICU admission period was significantly shorter and the rate of change in the Functional Independent Measure (FIM) was smaller than in the standard intervention group. The FIM upon discharge of the early intervention group was better than that of the standard intervention group. The results also demonstrated the beneficial effects of early physiotherapy on ADL and the ICU admission period.

Older people are at more risk from COVID-19 because of how the immune system ages. As people aging, their innate and adaptive immune responses change. Monocytes from older individuals produce less interferon in response to viral infection. They have a harder time killing infected cells and signaling the adaptive immune response to get going. Low-grade chronic inflammation in individuals that commonly occurs during aging can also dull the ability of the innate and adaptive immune responses to react to pathogens, giving the virus the upper hand. Viruses can take advantage of the immune system's slow start resulting in serious disease and death [11].

The amount of physical activity that a person does influences his/her risk of infection, most likely by affecting immune function. It is known that regular moderate exercise reduces the risk of infection compared with sedentary, but very prolonged bouts of exercise and periods of intensified training are associated with increased infection risk [12].

\section{Aim}

This study aims to recommend physiotherapy management program for prevention, decrease morbidity and mortality in COVID-19 patients and patients with other respiratory co-morbidities.

\section{Justification of the study:}

Physiotherapy may be beneficial in the respiratory treatment and physical rehabilitation of patients with COVID-19. This document has been prepared to provide information to physiotherapists and healthcare facilities about the po- 
tential role of physiotherapy in the management of COVID-19 patients and patients with other respiratory co-morbidities.

\section{Possible effect of exercises on COVID-19:}

Table 1 explored various changes in different systems of the body with respect to the physiological mechanisms involved. The physiotherapists usually aware about the effects of physical exercises training on various systems and realize the qualitative changes in the body for better performance.

Table 2 documented physiological effects of slow breathing techniques according to research in healthy humans and focuses on the physiological implications to the respiratory, cardiovascular, and cardiorespiratory systems, with particular focus on diaphragm activity, ventilation efficiency, haemodynamics, heart rate variability, cardiorespiratory coupling and respiratory sinus arrhythmia.

Implication of the study:

While this study suggests applying exercise program to geriatric people and

Table 1. Physiological effects of physical training [7].

\begin{tabular}{|c|c|}
\hline \multicolumn{2}{|c|}{ Physiological effects of physical training } \\
\hline Respiratory system & Circulatory system \\
\hline $\begin{array}{l}\text { - Lung volume increases as a result } \\
\text { of increase in vital capacity. } \\
\text { - Breathing frequency is decreased } \\
\text { in trained individuals. } \\
\text { - Maximum minute ventilation is } \\
\text { increased after training. } \\
\text { - Tidal volume is also increased. } \\
\text { - Physical exercises enhance venti- } \\
\text { latory efficiency. } \\
\text { - Diffusion capacity (gases exchange } \\
\text { capacity) is increased. }\end{array}$ & $\begin{array}{l}\text { - Internal size of the left ventricle increases. } \\
\text { - The contraction ability or strength of the heart increases. } \\
\text { - Resting heart rate decreases considerably. } \\
\text { - Maximum heart rate, either remains unchanged or } \\
\text { decreases slightly. } \\
\text { - The heart rate recovery period decreases. } \\
\text { - Blood volume increases as a result of increase in plasma } \\
\text { volume and RBC counts. } \\
\text { - Blood viscosity (thickness) decreases resulting good } \\
\text { - Birculation and oxygen availability. } \\
\text { - effective blood redistribution and increased capillarization. } \\
\text { - Reduction in resting blood pressure (both systolic and } \\
\text { diastolic). }\end{array}$ \\
\hline
\end{tabular}

Table 2. Physiological effects of slow breathing exercises [13].

\begin{tabular}{|c|c|c|}
\hline \multicolumn{3}{|c|}{ Physiological effects of slow breathingexercises } \\
\hline Respiratory system & Cardiovascular system & Cardiorespiratory system \\
\hline excursion & - Causes blood pressure pulse fluctuations to & - Improves pulmonary gas exchange efficiency \\
\hline - Enhances ventilation efficiency and arterial & synchronize with heart beat rhythm & - minimises cardiac work \\
\hline oxygenation via alveolar recruitment, and & - Synchronization of vasomotion & - buffers blood pressure fluctuations \\
\hline $\begin{array}{l}\text { distension and reduction of alveolar dead } \\
\text { space }\end{array}$ & $\begin{array}{l}\text { - May entrain and enhance vasomotion (and } \\
\text { microflow), i.e. to improve blood oxygenation }\end{array}$ & $\begin{array}{l}\text { - Clustering of heartbeats within inspiratory } \\
\text { phase (cardiorespiratory coupling) }\end{array}$ \\
\hline - Moderates chemoreflex sensitivity & $\begin{array}{l}\text { - Increases HRV and blood pressure fluctuations } \\
\text { - May decrease mean blood pressure }\end{array}$ & $\begin{array}{l}\text { - Synchronization of pulse harmonics of blood } \\
\text { flow and heart rhythm }\end{array}$ \\
\hline
\end{tabular}

LF = low frequency, HRV = Heart Rate Variability, baroreflex $=$ baroreceptor reflex, RSA = Respiratory Sinus Arrhythmia. 
other ages with other co-morbidities, there were important questions needed to be answered: Does the patient can do these exercises? Is it sure that the patient can do it safely? What about bed ridden patients and disabled patients?

The answer of all of these questions is patient safety, it is the main issue to confirm by close adherence of accompanying, care giver, nurse or physiotherapist each of them according needs and individual case, moreover, the exercises should be performed gently as he/she can only in normal body temperature for circulatory exercises by little modification can be applied in the kind of exercise, the way and position of performance and the repetitions that can be done with no exhaustion

\section{Suggested Exercises Protocol}

\section{I) Breathing exercises: (Table 3)}

\section{A) Pursed-lips breathing}

Pursed-lips breathing can slow down the breathing, reducing the work of breathing by keeping the airways open longer. This makes it easier for the lungs to function and improves the exchange of oxygen and carbon dioxide.

The patient will be instructed to do the exercise as following:

- Inhale slowly through your nostrils.

- Purse your lips, as if pouting or about to blow on something.

- Breathe out as slowly as possible through pursed lips. This should take at least twice as long as it did to breathe in.

\section{B) Incentive spirometer exercise:}

This will help the patient to breathe deeply and cough, thus clear the airway regularly, it gently exercise the lungs and aid in keeping the lungs as healthy as possible. The device helps retrain your lungs how to take slow and deep breaths. An incentive spirometer helps increase lung capacity and improves patients' ability to breathe.

Incentive spirometer device Figure 2, the user will be instructed as the following:

1) Close the lips tightly around the mouthpiece.

2) Breathe in slowly and deeply through the mouthtrying to raise the all balls to the highest level.

3) Hold the breath for 3 to 6 seconds trying to keep the balls at the highest level.

4) Release the mouthpiece and breathe out slowly.

5) Cough to help clear mucous from the throat and chest.

Cough deeply from your belly, not just from your throat. If you had surgery (chest or abdomen), first brace the area with a pillow.

Note: this device is a personal device and the user must not share with anybody else.

\section{C) Blow out the candle exercise:}

The aim of this exercise is to help to move from a more upper chest breathing pattern to one that uses the full capacity of the lungs. When under stress the 
Table 3. Suggested checklist schedule of breathing exercises.

\begin{tabular}{lllllllll}
\hline \multicolumn{7}{c}{ Checklist of daily breathing exercises } \\
\hline \multirow{7}{*}{ Day } & $9 \mathrm{am}$ & $11 \mathrm{am}$ & $1 \mathrm{pm}$ & $3 \mathrm{pm}$ & $5 \mathrm{pm}$ & $7 \mathrm{pm}$ & $9 \mathrm{pm}$ & $11 \mathrm{pm}$ \\
\cline { 2 - 6 } & & & & & & \\
\hline Sun & & & & \\
Mon & \\
Tue & \\
Wed & \\
Thu & \\
Fri & \\
Sat
\end{tabular}

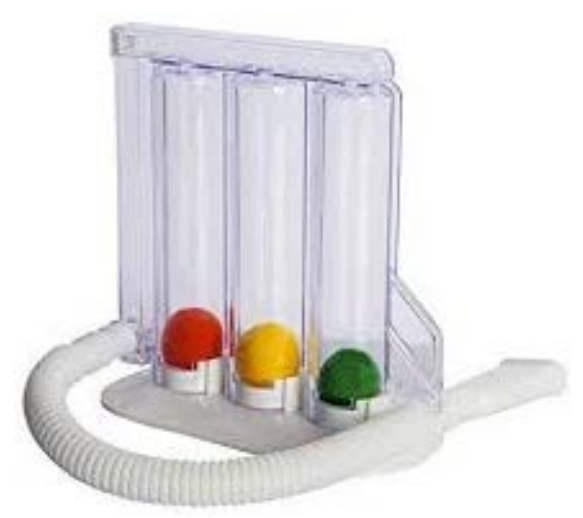

Figure 2. Incentive spirometer device.

person can move towards breathing into the top of our lungs, with little movement happening at the bottom of the ribcage. This means we use the muscles at the front of the neck more than maybe helpful.

This exercise is a preliminary exercise to become comfortable with before moving on to the other parts: Head Retraction and Brügger Exercises

The patient will be instructed to do the exercise as following:

1) Imagine a birthday candle.

2) Take in a deep breath through the nose

3) Then exhale through the mouth to blow out the candle trying empty your lungs as best you can. When we are chest breathing we can sometimes not empty the lungs as fully as we might. So with this exercise we are looking to focus on breathing out slowly and fully.

4) Pause at the end of the out breath. Once you have fully breathed out, close your lips and pause for a count of one.

5) Breathe in through your nose. You do not need to actually do anything special with your inhalation, just let it happen. Your body knows how to do it.

\section{Notes:}

This exercise is all about the exhalation and that is the focus, rather than 
doing anything fancy when you breathe in.

The breathing in this exercise should not be making you feel anxious, so if you start to feel odd or worried doing this exercise then (please) stop and go back to breathing as you normally would.

II) Circulatory exercise: (Table 4)

(From sitting/long sitting/lying position)

1) Fist pump

2) Bend elbow

3) Forward raise of shoulders

4) Horizontal shoulders abduction (bilateral)

5) Trunk twist

6) Ankle pump

7) Knee extension

8) Hip flexion with flexed knee

The patient will be instructed the following:

- To do these exercises alternatively limb by limb (except horizontal shoulder abduction)

- Do these exercises gently

- Moderate speed as much as he/she can

- If the patient can't do any above exercise, try to do it from lying position otherwise he/she shouldn't be pushed to do it

- Avoid exhaustion

- If the normal geriatric/patient is dependent, must be under supervision

- Safety first, don't permit unacceptable shortness of breathing or raising in heart rate

- Apply adequate rest time between exercises.

\section{Application of the Protocol in Different COVID-19 Stages}

\section{Stage (A) prophylactic stage:}

Target population: normal geriatrics and patients with comorbidities like cardiac

Table 4. Suggested checklist schedule of circulatory exercises.

\begin{tabular}{|c|c|c|c|}
\hline \multicolumn{4}{|c|}{ Checklist of daily circulatory exercises } \\
\hline \multirow{2}{*}{ Day } & \multicolumn{3}{|c|}{ Parts of day } \\
\hline & Morning & Afternoon & Evening \\
\hline \multicolumn{4}{|l|}{ Sun } \\
\hline \multicolumn{4}{|l|}{ Mon } \\
\hline \multicolumn{4}{|l|}{ Tue } \\
\hline \multicolumn{4}{|l|}{ Wed } \\
\hline \multicolumn{4}{|l|}{ Thu } \\
\hline \multicolumn{4}{|l|}{ Fri } \\
\hline Sat & & & \\
\hline
\end{tabular}


patients, diabetics, other chest patients etc.

Mode: self-dependent exercises

Checklist responsibility: the person him/herself

Intensity: no resistance

Repetitions: 10 times per set, one set is enough for each exercise in the check box as can as possible.

\section{Stage (B) home/quarantine stage:}

Target population: suspected/early stage infection of Covid-19 in geriatrics and patients with comorbidities like cardiac patients, diabetics, other chest patients etc.

Mode: self-dependent exercises under supervision of accompanying/health care giver.

Checklist responsibility: patient's accompanying/care giver

Intensity: no resistance

Repetitions: 10 times per set, one set is enough for each exercise in the check box as can as possible

\section{Stage (C) intensive care unit (ICU) stage:}

Target population: infection of COVID-19 in geriatrics and patients with comorbidities like cardiac patients, diabetics, other chest patients etc. in ICU stage

Mode: responsibility of specialized physical therapist

Checklist responsibility: physical therapist

Intensity: active respiratory exercises, active assisted circulatory exercises if the patient can't do the exercises alone, passive circulatory exercises in case of fever

Repetitions: 5 - 7 times per set, one set is enough for each exercise in the check box as can as possible

\section{Discussion}

Three pandemics occurred in the previous century: "Spanish influenza" in 1918, "Asian influenza" in 1957, and "Hong Kong influenza" in 1968 [14]. Over the last decades the emergence of SARS, H1N1, Influenza A, and Middle Eastern Respiratory Syndrome (MERS) illustrates the powerful threat of cross-species transmission events that have led to novel human disease with consequential global impact [15]. Coronaviruses are important human and animal pathogens. At the end of 2019, a novel coronavirus was identified as the cause of a cluster of pneumonia cases in Wuhan, a city in the Hubei Province of China. It rapidly spread, resulting in an epidemic throughout China, followed by an increasing number of cases in other countries throughout the world [16].

Mostly, recent scientific articles in physical therapy field that interfere with COVID-19 management talked about role of physiotherapist in chest clear on percentages of patients with chest secretions and patients with co-morbid that have another health issues that require physical therapy service e.g. neurological issue. They neglected the role of exercises even gentle in improving cardiopul- 
monary system, improving the quality of breathing, raising the immune of lungs and increase self-confidence and sense of well-being. On the other hand, however physical therapy should be recognized as an effective treatment method that improves the prognosis associated with pulmonary complications which decrease mortality, it can provide protective umbrella from morbidity of COVID19 [17].

Simply, coronavirus proliferation in lung decreases the capacity of ventilation and the quality of breathing. The goal we need is to strengthen the immune system or reducing the ability of the virus to spread across the pulmonary tissue may be achieved by increasing chest expansion, the respiratory muscles power especially the diaphragm and encouraging effective passive recoil of it during quite breathing, this is what happened as physiological adaptation to breathing exercise through direct respiratory muscles activity during breathing exercises and through indirect respiratory system activity as response of physiological oxygen demand during circulatory exercises, these theories are compatible with New England Complex System Institute (March 2020) which recommended aerobic exercise before infection to strengthen cardiovascular health. Once infected, during the period of mild symptoms, moderate daily aerobic exercise can improve lung ventilation. Such exercise may benefit immune function as well [8].

There is a need to more fully incorporate multidisciplinary rehabilitation teams inclusive of physiotherapy along the coronavirus disease from acute and inpatient care, through to the ambulatory settings and onwards into the community [15]. To be effective, rehabilitative interventions must be considered during the planning and allocation of resources used to control a disease pandemic [18].

This article is compatible with Kusnanto et al. (2018) [19] spiritual emotional breathing (SEB) can improve the quality of respiratory function and the modulation of immune response in tuberculosis patients. The emotional spiritual approach is part of the science of energy psychology that aims to turn the negative energy in a person into positive energy that can help the healing process. This therapy is performed as a complementary therapy for TB patients to improve their quality of life and the control of symptoms.

\section{Limitation:}

Given the recent presentation of COVID-19, clinical guidance may change as more is learnt about the natural history of this disease.

\section{Conclusions and Recommendations}

"WHO has been assessing this outbreak around the clock and we are deeply concerned both by the alarming levels of spread and severity, and by the alarming levels of inaction" WHO Director-General said at the COVID-19 media briefing in $11^{\text {th }}$ of March 2020 [20].

Pneumonia is one of its common complications which increase mortality rate in geriatrics and patients with other co-morbidities, breathing and circulatory 
exercises have great effects on decreasing pneumonia, improving pulmonary function, increasing lung immunity and improving psychological status that helping patients to protect high risk patients and may be a cause of survive. This study recommended the following:

- Application of this protocol in treatment program of COVID-19.

- Physiotherapists are required to have specialized knowledge, skills and decision-making to work within ICU.

- Further researches are needed to assess the role of exercises in prevention and management of COVID-19.

\section{Conflicts of Interest}

The authors declare no conflicts of interest regarding the publication of this paper.

\section{References}

[1] CDC COVID-19 Response Team (2020) Severe Outcomes among Patients with Coronavirus Disease 2019 (COVID-19)—United States, February 12-March 16, 2020. Morbidity Mortal Weekly Report (MMWR), 69, 343-346. https://doi.org/10.15585/mmwr.mm6912e2

[2] Huang, C., et al. (2020) Clinical Features of Patients Infected with Novel Coronavirus in Wuhan, China. Lancet, 395, 497-506.

https://doi.org/10.1016/S0140-6736(20)30183-5

[3] WHO (2020) Coronavirus. https://www.who.int/health-topics/coronavirus\#tab=tab_1

[4] Tse, G.M., To, K.F., Chan, P.K., et al. (2004) Pulmonary Pathological Features in Coronavirus Associated Severe Acute Respiratory Syndrome (SARS). Journal of Clinical Pathology, 57, 260-265. https://doi.org/10.1136/jcp.2003.013276

[5] Zheng, Y., et al. COVID-19 and the Cardiovascular System. Nature Reviews Cardiology, 17, 259-260. http://www.nature.com/nrcardio

[6] Nystoriak, M. and Bhatnagar, A. (2018) Cardiovascular Effects and Benefits of Exercise. Frontiers in Cardiovascular Medicine, 6, 135.

https://doi.org/10.3389/fcvm.2018.00135

[7] Gulam, A. (2016) Impact of Exercises on Muscular, Respiratory and Circulatory Body Systems. International Journal of Academic Research and Development, 1 , 59-62.

[8] Elias, B., Shen, C. and Bar-Yam, Y. (2020) Respiratory Health for Better COVID-19 Outcomes. New England Complex Systems Institute.

[9] World Health Organization (2020) Mental Health and Psychosocial Considerations during COVID-19 Outbreak.

[10] Chigira, Y., Takai, T., Igusa, H. and Dobashi, K. (2015) Effects of Early Physiotherapy with Respect to Severity of Pneumonia of Elderly Patients Admitted to an Intensive Care Unit: A Single Center Study in Japan. Journal of Physical Therapy Science, 27, 2053-2056. https://doi.org/10.1589/jpts.27.2053

[11] Geiss, B. Older People Are at More Risk from COVID-19 Because of How the immune System Ages. (Cited at 2 July 2020) https://theconversation.com/older-people-are-at-more-risk-from-covid-19-because -of-how-the-immune-system-ages-133899 
[12] Gleeson, M. (2015) Effect of Exercise on Immune Function. Sports Science EXchange, 28, 1-6.

[13] Russo, M.A., Santarelli, D.M. and O’Rourke, D. (2017) The Physiological Effects of Slow Breathing in the Healthy Human. Breathe, 13, 299-308. https://doi.org/10.1183/20734735.009817

[14] Awadalla, H.I. and El-Khouly, N.F. (2014) Human Pandemic Threat by $\mathrm{H}_{5} \mathrm{~N}_{1}$ (Avian Influenza). African Journal of Microbiology Research, 8, 406-410. https://doi.org/10.5897/AJMR10.303

[15] Landry, M.D., Landry, M.D., Geddes, L., et al. (2020) Early Reflection on the Global Impact of COVID19, and Implications for Physiotherapy. Physiotherapy, 107, A1-A3. https://doi.org/10.1016/j.physio.2020.03.003

[16] She, J., Jiang, J., Ye, L., Hu, L., Bai, C. and Song, Y. (2020) 2019 Novel Coronavirus of Pneumonia in Wuhan, China: Emerging Attack and Management Strategies. Clinical and Translational Medicine, 9, e19. https://doi.org/10.1186/s40169-020-00271-Z

[17] Physiopedia Contributors (2020) Role of the Physiotherapist in COVID-19. https://www.physio-pedia.com/index.php?title=Role_of_the_Physiotherapist_in_C OVID-19\&oldid $=239889$

[18] Xiang, Y.-T., Yang, Y., Li, W., Zhang, L., Zhang, Q. and Cheung, T. (2020) Timely Mental Health Care for the 2019 Novel Coronavirus Outbreak Is Urgently Needed. Lancet Psychiatry, 7, 228-229. https://doi.org/10.1016/S2215-0366(20)30046-8

[19] Kusnanto, K., Haryanto, J., Sukartini, T., Ulfiana, E. and Putra, M.M. (2018) The Effectiveness of Spiritual Emotional Breathing towards Respiratory Function and Immune Response of Tuberculosis Patients. Jurnal Ners, 13, 93-97. https://doi.org/10.20473/jn.v13i1.8373

[20] WHO. WHO Director-General's Opening Remarks at the Media Briefing on COVID19-11 May 2020.

https://www.who.int/dg/speeches/detail/who-director-general-s-opening-remarks-a t-the-media-briefing-on-covid-19---11-march-2020 